\title{
Congruences for Stirling numbers and Eulerian numbers
}

by

\section{Hui-Qin CAO and HaO PAN (Nanjing)}

1. Introduction. As usual, we set $\left(\begin{array}{l}x \\ 0\end{array}\right)=1$ and

$$
\left(\begin{array}{l}
x \\
k
\end{array}\right)=\frac{x(x-1) \cdots(x-k+1)}{k !} \quad \text { for } k=1,2, \ldots .
$$

We also set $\left(\begin{array}{l}x \\ k\end{array}\right)=0$ for any negative integer $k$.

Let $p$ be a prime, and let $n>0$ and $r$ be integers. In 1913, A. Fleck (cf. $[3$, p. 274]) discovered that

$$
\sum_{k \equiv r(\bmod p)}\left(\begin{array}{l}
n \\
k
\end{array}\right)(-1)^{k} \equiv 0\left(\bmod p^{\left\lfloor\frac{n-1}{p-1}\right\rfloor}\right),
$$

where $\lfloor\cdot\rfloor$ is the floor function. In 1977, C. S. Weisman [14] extended Fleck's congruence to prime power moduli in the following way:

$$
\sum_{k \equiv r\left(\bmod p^{\alpha}\right)}\left(\begin{array}{l}
n \\
k
\end{array}\right)(-1)^{k} \equiv 0\left(\bmod p^{\left\lfloor\frac{n-p^{\alpha-1}}{p^{\alpha-1}(p-1)}\right\rfloor}\right),
$$

where $\alpha$ is a positive integer and $n \geq p^{\alpha-1}$.

In 2005, in his lecture notes on Fontaine's rings, D. Wan got another extension of Fleck's congruence:

$$
\sum_{k \equiv r(\bmod p)}\left(\begin{array}{l}
n \\
k
\end{array}\right)(-1)^{k}\left(\begin{array}{c}
(k-r) / p \\
l
\end{array}\right) \equiv 0\left(\bmod p^{\left\lfloor\frac{n-l p-1}{p-1}\right\rfloor}\right),
$$

where $l \in \mathbb{N}=\{0,1,2, \ldots\}$ and $n>l p$. Later, by a combinatorial approach, Z. W. Sun [7] established a common generalization of Weisman's and Wan's extensions of Fleck's congruence:

2000 Mathematics Subject Classification: Primary 11A07; Secondary 05A15, 11B65, $11 \mathrm{~B} 73$.

Key words and phrases: Stirling number, Eulerian number, congruence. 


$$
\begin{aligned}
\operatorname{ord}_{p}\left(\sum_{k \equiv r\left(\bmod p^{\beta}\right)}\left(\begin{array}{l}
n \\
k
\end{array}\right)(-1)^{k}\left(\begin{array}{c}
\left\lfloor(k-r) / p^{\alpha}\right\rfloor \\
l
\end{array}\right)\right) \\
\quad \geq\left\lfloor\frac{n-p^{\alpha-1}-l}{p^{\alpha-1}(p-1)}\right\rfloor-(l-1) \alpha-\beta
\end{aligned}
$$

provided that $\alpha, \beta \in \mathbb{N}, \alpha \geq \beta$ and $n \geq p^{\alpha-1}$, where $\operatorname{ord}_{p}(a)=\sup \{i \in \mathbb{N}$ : $\left.p^{i} \mid a\right\}$ is the $p$-adic order of $a \in \mathbb{Z}$.

In fact, with the help of the $\psi$-operator in Fontaine's theory of $(\phi, \Gamma)$ modules, (1.3) and (1.4) can be improved as follows [13]:

$$
\operatorname{ord}_{p}\left(\sum_{k \equiv r\left(\bmod p^{\alpha}\right)}\left(\begin{array}{l}
n \\
k
\end{array}\right)(-1)^{k}\left(\begin{array}{c}
(k-r) / p^{\alpha} \\
l
\end{array}\right)\right) \geq\left\lfloor\frac{n-p^{\alpha-1}-l p^{\alpha}}{p^{\alpha-1}(p-1)}\right\rfloor
$$

A combinatorial proof of (1.5) is given in [11]. On the other hand, motivated by algebraic topology, D. M. Davis and Z. W. Sun $[2,10]$ showed that

$$
\operatorname{ord}_{p}\left(\sum_{k \equiv r\left(\bmod p^{\alpha}\right)}\left(\begin{array}{l}
n \\
k
\end{array}\right)(-1)^{k}\left(\begin{array}{c}
(k-r) / p^{\alpha} \\
l
\end{array}\right)\right) \geq \operatorname{ord}_{p}\left(\left\lfloor\frac{n}{p^{\alpha}}\right\rfloor !\right)
$$

and

$$
\begin{aligned}
\operatorname{ord}_{p}\left(\sum_{k \equiv r\left(\bmod p^{\alpha}\right)}\left(\begin{array}{l}
n \\
k
\end{array}\right)(-1)^{k}\right. & \left.\left(\begin{array}{c}
(k-r) / p^{\alpha} \\
l
\end{array}\right)\right) \\
& \geq \operatorname{ord}_{p}\left(\left\lfloor\frac{n}{p^{\alpha-1}}\right\rfloor !\right)-l-\operatorname{ord}_{p}(l !) .
\end{aligned}
$$

Notice that (1.6) and (1.7) cannot be deduced from (1.5) though they have the similar flavor. For the further developments on (1.5) and (1.6), the reader is referred to $[8,11,12,9]$.

In the present paper, we shall investigate some Fleck-Weisman and Davis-Sun type congruences for other number arrays. The Stirling number $s(n, k)$ of the first kind is the number of permutations of $\{1, \ldots, n\}$ which contain exactly $k$ permutation cycles. $s(n, k)(0 \leq k \leq n)$ can be given by

$$
x(x+1) \cdots(x+n-1)=\sum_{k=0}^{n} s(n, k) x^{k} .
$$

Similarly, the Stirling number $S(n, k)$ of the second kind is the number of ways to partition a set of cardinality $n$ into $k$ nonempty subsets. It is well known that

$$
x^{n}=\sum_{k=0}^{n} S(n, k) k !\left(\begin{array}{l}
x \\
k
\end{array}\right)
$$

for $n \in \mathbb{N}$. In particular, we set $s(0,0)=S(0,0)=1$ and $s(n, k)=S(n, k)$ $=0$ whenever $k>n$. 
Another important array of numbers related to permutations is formed by the so-called Eulerian numbers. For an arbitrary permutation $\pi=a_{1} \cdots a_{n}$ of $\{1, \ldots, n\}$, we say that an element $i \in\{1, \ldots, n-1\}$ is an ascent of $\pi$ if $a_{i}<a_{i+1}$. The Eulerian number $\left\langle\begin{array}{l}n \\ k\end{array}\right\rangle$ is the number of permutations of $\{1, \ldots, n\}$ having exactly $k$ ascents. (Another commonly used notation is $A(n, k)$ (sometimes $A_{n, k}$ ) with $A(n, k)=\left\langle\begin{array}{c}n \\ k-1\end{array}\right\rangle$.) Clearly $\left\langle\begin{array}{l}n \\ 0\end{array}\right\rangle=1$ and $\left\langle\begin{array}{l}n \\ k\end{array}\right\rangle=0$ for every $k>n-1$. We also set $\left\langle\begin{array}{l}n \\ k\end{array}\right\rangle=0$ when $k<0$. It is easy to check that the Eulerian numbers satisfy the recurrence relation

$$
\left\langle\begin{array}{l}
n \\
k
\end{array}\right\rangle=(k+1)\left\langle\begin{array}{c}
n-1 \\
k
\end{array}\right\rangle+(n-k)\left\langle\begin{array}{l}
n-1 \\
k-1
\end{array}\right\rangle .
$$

The Stirling numbers of the first and second kind and the Eulerian numbers play important roles in enumerative combinatorics (cf. [4, pp. 257-272] and [5, pp. 123-127]). Many arithmetic properties of these numbers are listed in [6, Chapter 5]. A little surprisingly, the Eulerian numbers satisfy a congruence mixing (1.5) and (1.7) in some way.

TheOREM 1.1. Let $p$ be a prime. Let $n>0$ and $r$ be integers. Then for any positive integer $\alpha$ and $l \in \mathbb{N}$, we have

$$
\begin{aligned}
\operatorname{ord}_{p}\left(\sum_{k \equiv r\left(\bmod p^{\alpha}\right)}\left\langle\begin{array}{l}
n \\
k
\end{array}\right\rangle\left(\begin{array}{c}
(k-r) / p^{\alpha} \\
l
\end{array}\right)\right) \\
\quad \geq \operatorname{ord}_{p}\left(\left\lfloor\frac{n}{p^{\alpha-1}}\right\rfloor !\right)-l-\left\lceil\frac{1+l}{p-1}\right\rceil,
\end{aligned}
$$

where $\lceil\cdot\rceil$ is the ceiling function. Moreover, if $a$ is an integer with $a \equiv$ $1(\bmod p)$, then

$$
\sum_{k \equiv r\left(\bmod p^{\alpha}\right)}\left\langle\begin{array}{l}
n \\
k
\end{array}\right\rangle a^{k} \equiv 0\left(\bmod p^{\operatorname{ord}_{p}\left(\left\lfloor n / p^{\alpha-1}\right\rfloor !\right)-1}\right)
$$

provided that $n \geq p^{\alpha}$.

The results on Stirling numbers are a little complicated. We have the following Davis-Sun type congruence:

TheOrem 1.2. Let $p$ be a prime and $n, m$ be positive integers. For arbitrary integers $a$ and $r$,

$$
\operatorname{ord}_{p}\left(\sum_{k \equiv r(\bmod p-1)} s(n, k) S(k, m) a^{k}\right) \geq \operatorname{ord}_{p}(n !)-\operatorname{ord}_{p}(m !) .
$$

Moreover, if $f(x)$ is a polynomial with integral coefficients, then

$$
\operatorname{ord}_{p}\left(\sum_{k \equiv r(\bmod p-1)} s(n, k) f(k) a^{k}\right) \geq \operatorname{ord}_{p}(n !)-\log _{p}\left(\begin{array}{l}
n \\
l
\end{array}\right),
$$

where $l=\min \{\operatorname{deg} f,\lfloor n / p\rfloor\}$ and $\log _{p} x=\log x / \log p$. 
Also, we have the following Weisman type congruence.

TheOREM 1.3. Let $p$ be a prime and $n, m$ be positive integers. For any integers $a, r$ and $\alpha \geq 1$,

$$
\begin{aligned}
\operatorname{ord}_{p}\left(\sum_{k \equiv r\left(\bmod p^{\alpha}(p-1)\right)} s(n, k) S(k, m) a^{k}\right) & \\
& \geq\left\lfloor\frac{n-p^{\alpha}}{p^{\alpha}(p-1)}\right\rfloor-\operatorname{ord}_{p}(m !) .
\end{aligned}
$$

Combining Theorems 1.2 and 1.3, we immediately deduce

Corollary 1.1.

$$
\operatorname{ord}_{p}\left(\sum_{k \equiv r\left(\bmod \varphi\left(p^{\alpha}\right)\right)} s(n, k) a^{k}\right) \geq \begin{cases}\operatorname{ord}_{p}(n !) & \text { if } \alpha=1, \\ \left\lfloor\left(n-p^{\alpha-1}\right) / \varphi\left(p^{\alpha}\right)\right\rfloor & \text { if } \alpha \geq 2,\end{cases}
$$

where $\varphi$ denotes the Euler totient function.

The proofs of Theorems 1.1-1.3 will be given in the next sections.

2. Congruences for Eulerian numbers: $k \equiv r\left(\bmod p^{\alpha}\right)$. In this section, we shall prove Theorem 1.1. The following lemma gives a weak (but non-trivial) lower bound for the $p$-adic order of $S(n, k)$.

Lemma 2.1. Let $p$ be a prime and let $n, k \in \mathbb{N}$. Then for any positive integer $\alpha$,

$$
\operatorname{ord}_{p}(k ! S(n, k)) \geq \operatorname{ord}_{p}\left(\left\lfloor\frac{n}{p^{\alpha-1}}\right\rfloor !\right)-\left\lfloor\frac{n-k}{p^{\alpha-1}(p-1)}\right\rfloor .
$$

Proof. We use induction on $n$. There is nothing to do when $n=0$. Below we assume that $n \geq 1$ and (2.1) is valid for smaller values of $n$. Obviously (2.1) holds for $k=0$ since

$$
\operatorname{ord}_{p}\left(\left\lfloor\frac{n}{p^{\alpha-1}}\right\rfloor !\right)=\sum_{i=\alpha}^{\infty}\left\lfloor\frac{n}{p^{i}}\right\rfloor<\sum_{i=\alpha}^{\infty} \frac{n}{p^{i}}=\frac{n}{p^{\alpha-1}(p-1)} .
$$

Suppose that $k \geq 1$. It is known (cf. [1, p. 209]) that

$$
k ! S(n, k)=\sum_{i=k-1}^{n-1}\left(\begin{array}{l}
n \\
i
\end{array}\right)(k-1) ! S(i, k-1) \quad(k \geq 1) .
$$

Observe that 


$$
\begin{aligned}
\operatorname{ord}_{p}\left(\left(\begin{array}{c}
n \\
i
\end{array}\right)\right) & =\sum_{j=1}^{\infty}\left(\left\lfloor\frac{n}{p^{j}}\right\rfloor-\left\lfloor\frac{n-i}{p^{j}}\right\rfloor-\left\lfloor\frac{i}{p^{j}}\right\rfloor\right) \\
& \geq \sum_{j=\alpha}^{\infty}\left(\left\lfloor\frac{n}{p^{j}}\right\rfloor-\left\lfloor\frac{n-i}{p^{j}}\right\rfloor-\left\lfloor\frac{i}{p^{j}}\right\rfloor\right) \\
& =\operatorname{ord}_{p}\left(\left\lfloor\frac{n}{p^{\alpha-1}}\right\rfloor !\right)-\operatorname{ord}_{p}\left(\left\lfloor\frac{n-i}{p^{\alpha-1}}\right\rfloor !\right)-\operatorname{ord}_{p}\left(\left\lfloor\frac{i}{p^{\alpha-1}}\right\rfloor !\right) .
\end{aligned}
$$

By the induction hypothesis, for $k-1 \leq i \leq n-1$ we have

$$
\begin{aligned}
& \operatorname{ord}_{p}\left(\left(\begin{array}{l}
n \\
i
\end{array}\right)(k-1) ! S(i, k-1)\right) \\
& \geq \operatorname{ord}_{p}\left(\left(\begin{array}{c}
n \\
i
\end{array}\right)\right)+\operatorname{ord}_{p}\left(\left\lfloor\frac{i}{p^{\alpha-1}}\right\rfloor !\right)-\left\lfloor\frac{i-(k-1)}{p^{\alpha-1}(p-1)}\right\rfloor \\
& \geq \operatorname{ord}_{p}\left(\left\lfloor\frac{n}{p^{\alpha-1}}\right\rfloor !\right)-\operatorname{ord}_{p}\left(\left\lfloor\frac{n-i}{p^{\alpha-1}}\right\rfloor !\right)-\left\lfloor\frac{i-k+1}{p^{\alpha-1}(p-1)}\right\rfloor \\
& \geq \operatorname{ord}_{p}\left(\left\lfloor\frac{n}{p^{\alpha-1}}\right\rfloor !\right)-\left\lfloor\frac{n-i-1}{p^{\alpha-1}(p-1)}\right\rfloor-\left\lfloor\frac{i-k+1}{p^{\alpha-1}(p-1)}\right\rfloor \\
& \geq \operatorname{ord}_{p}\left(\left\lfloor\frac{n}{p^{\alpha-1}}\right\rfloor !\right)-\left\lfloor\frac{n-k}{p^{\alpha-1}(p-1)}\right\rfloor \text {. }
\end{aligned}
$$

LEMMA 2.2. Let $n$ be a positive integer. Then for any polynomial $f(x) \in$ $\mathbb{Q}[x]$ we have

$$
\sum_{k}\left\langle\begin{array}{l}
n \\
k
\end{array}\right\rangle f(k) x^{k}=\sum_{m} m ! S(n, m) \sum_{i=0}^{n-m}\left(\begin{array}{c}
n-m \\
i
\end{array}\right)(-1)^{n-m-i} f(i) x^{i} .
$$

Proof. It is sufficient to prove (2.3) for $f(x)=x^{l}, l \in \mathbb{N}$. In the case $l=0,(2.3)$ reduces to

$$
\begin{aligned}
\sum_{k}\left\langle\begin{array}{l}
n \\
k
\end{array}\right\rangle x^{k} & =\sum_{m} m ! S(n, m) \sum_{i=0}^{n-m}\left(\begin{array}{c}
n-m \\
i
\end{array}\right)(-1)^{n-m-i} x^{i} \\
& =\sum_{m} m ! S(n, m)(x-1)^{n-m},
\end{aligned}
$$

which is true (cf. [4, p. 269]). Now assume that $l>0$ and (2.3) holds for $l-1$, that is,

$$
\sum_{k}\left\langle\begin{array}{l}
n \\
k
\end{array}\right\rangle k^{l-1} x^{k}=\sum_{m} m ! S(n, m) \sum_{i=0}^{n-m}\left(\begin{array}{c}
n-m \\
i
\end{array}\right)(-1)^{n-m-i} i^{l-1} x^{i} .
$$


Taking derivatives of both sides of the above equation with respect to $x$, we get

$$
\sum_{k}\left\langle\begin{array}{l}
n \\
k
\end{array}\right\rangle k^{l} x^{k-1}=\sum_{m} m ! S(n, m) \sum_{i=1}^{n-m}\left(\begin{array}{c}
n-m \\
i
\end{array}\right)(-1)^{n-m-i} i^{l} x^{i-1} .
$$

Proof of (1.8). Let $\zeta$ be a primitive $p^{\alpha}$ th root of the unity. Then

$$
\begin{aligned}
\sum_{k \equiv r\left(\bmod p^{\alpha}\right)}\left\langle\begin{array}{l}
n \\
k
\end{array}\right\rangle\left(\begin{array}{c}
(k-r) / p^{\alpha} \\
l
\end{array}\right) & =\sum_{k}\left\langle\begin{array}{l}
n \\
k
\end{array}\right\rangle\left(\begin{array}{c}
(k-r) / p^{\alpha} \\
l
\end{array}\right) \frac{1}{p^{\alpha}} \sum_{j=0}^{p^{\alpha}-1} \zeta^{j(k-r)} \\
& =\frac{1}{p^{\alpha}} \sum_{j=0}^{p^{\alpha}-1} \zeta^{-j r} \sum_{k}\left\langle\begin{array}{l}
n \\
k
\end{array}\right\rangle\left(\begin{array}{c}
(k-r) / p^{\alpha} \\
l
\end{array}\right) \zeta^{j k} .
\end{aligned}
$$

Note that $\left(\begin{array}{c}(x-r) / p^{\alpha} \\ l\end{array}\right)$ is a polynomial in $x$ with rational coefficients of degree $l$. By Lemma 2.2, we have

$$
\begin{aligned}
& \sum_{k \equiv r\left(\bmod p^{\alpha}\right)}\left\langle\begin{array}{c}
n \\
k
\end{array}\right\rangle\left(\begin{array}{c}
(k-r) / p^{\alpha} \\
l
\end{array}\right) \\
& \quad=\frac{1}{p^{\alpha}} \sum_{j=0}^{p^{\alpha}-1} \zeta^{-j r} \sum_{m} m ! S(n, m) \sum_{i=0}^{n-m}\left(\begin{array}{c}
n-m \\
i
\end{array}\right)(-1)^{n-m-i}\left(\begin{array}{c}
(i-r) / p^{\alpha} \\
l
\end{array}\right) \zeta^{j i} \\
& =\sum_{m=0}^{n} m ! S(n, m) \sum_{i=0}^{n-m}\left(\begin{array}{c}
n-m \\
i
\end{array}\right)(-1)^{n-m-i}\left(\begin{array}{c}
(i-r) / p^{\alpha} \\
l
\end{array}\right) \frac{1}{p^{\alpha}} \sum_{j=0}^{p^{\alpha}-1} \zeta^{j(i-r)} \\
& =\sum_{m=0}^{n} m ! S(n, m) \sum_{i \equiv r\left(\bmod p^{\alpha}\right)}\left(\begin{array}{c}
n-m \\
i
\end{array}\right)(-1)^{n-m-i}\left(\begin{array}{c}
(i-r) / p^{\alpha} \\
l
\end{array}\right) .
\end{aligned}
$$

Applying Lemma 2.1 and (1.5), for every $0 \leq m \leq n$ we have

$$
\begin{aligned}
\operatorname{ord}_{p}(m ! S(n, m) & \left.\sum_{i \equiv r\left(\bmod p^{\alpha}\right)}\left(\begin{array}{c}
n-m \\
i
\end{array}\right)(-1)^{n-m-i}\left(\begin{array}{c}
(i-r) / p^{\alpha} \\
l
\end{array}\right)\right) \\
& \geq \operatorname{ord}_{p}\left(\left\lfloor\frac{n}{p^{\alpha-1}}\right\rfloor !\right)-\left\lfloor\frac{n-m}{p^{\alpha-1}(p-1)}\right\rfloor+\left\lfloor\frac{n-m-p^{\alpha-1}-l p^{\alpha}}{p^{\alpha-1}(p-1)}\right\rfloor \\
& \geq \operatorname{ord}_{p}\left(\left\lfloor\frac{n}{p^{\alpha-1}}\right\rfloor !\right)-\left\lceil\frac{p^{\alpha-1}+l p^{\alpha}}{p^{\alpha-1}(p-1)}\right\rceil \\
& =\operatorname{ord}_{p}\left(\left\lfloor\frac{n}{p^{\alpha-1}}\right\rfloor !\right)-l-\left\lceil\frac{1+l}{p-1}\right\rceil .
\end{aligned}
$$


Proof of (1.9). Let $\zeta$ be a primitive $p^{\alpha}$ th root of the unity. Using (2.4) we have

$$
\begin{aligned}
\sum_{k \equiv r\left(\bmod p^{\alpha}\right)}\left\langle\begin{array}{l}
n \\
k
\end{array}\right\rangle a^{k} & =\sum_{k}\left\langle\begin{array}{l}
n \\
k
\end{array}\right\rangle a^{k} \frac{1}{p^{\alpha}} \sum_{j=0}^{p^{\alpha}-1} \zeta^{j(k-r)}=\frac{1}{p^{\alpha}} \sum_{j=0}^{p^{\alpha}-1} \zeta^{-j r} \sum_{k}\left\langle\begin{array}{l}
n \\
k
\end{array}\right\rangle\left(a \zeta^{j}\right)^{k} \\
& =\frac{1}{p^{\alpha}} \sum_{j=0}^{p^{\alpha}-1} \zeta^{-j r} \sum_{m} m ! S(n, m)\left(a \zeta^{j}-1\right)^{n-m} \\
& =\frac{1}{p^{\alpha}} \sum_{j=0}^{p^{\alpha}-1} \zeta^{-j r} \sum_{m=0}^{n} m ! S(n, m) \sum_{i=0}^{n-m}\left(\begin{array}{c}
n-m \\
i
\end{array}\right)(-1)^{n-m-i} a^{i} \zeta^{j i} \\
& =\sum_{m=0}^{n} m ! S(n, m)(-1)^{n-m} \sum_{i=0}^{n-m}\left(\begin{array}{c}
n-m \\
i
\end{array}\right)(-a)^{i} \frac{1}{p^{\alpha}} \sum_{j=0}^{p^{\alpha}-1} \zeta^{j(i-r)} \\
& =\sum_{m=0}^{n} m ! S(n, m)(-1)^{n-m} \sum_{i \equiv r\left(\bmod p^{\alpha}\right)}\left(\begin{array}{c}
n-m \\
i
\end{array}\right)(-a)^{i} .
\end{aligned}
$$

In view of Lemma 2.1 and (1.8) in [7],

$$
\begin{aligned}
\operatorname{ord}_{p}(m ! S(n, m) & \left.\sum_{i \equiv r\left(\bmod p^{\alpha}\right)}\left(\begin{array}{c}
n-m \\
i
\end{array}\right)(-a)^{i}\right) \\
\geq & \operatorname{ord}_{p}\left(\left\lfloor\frac{n}{p^{\alpha-1}}\right\rfloor !\right)-\left\lfloor\frac{n-m}{p^{\alpha-1}(p-1)}\right\rfloor+\left\lfloor\frac{n-m-p^{\alpha-1}}{p^{\alpha-1}(p-1)}\right\rfloor \\
\geq & \operatorname{ord}_{p}\left(\left\lfloor\frac{n}{p^{\alpha-1}}\right\rfloor !\right)-1 \quad \text { for every } 0 \leq m \leq n .
\end{aligned}
$$

3. Congruences for Stirling numbers: $k \equiv r(\bmod p-1)$. In this section, we shall prove Theorem 1.2. For a prime $p$, we let $\mathbb{Z}_{p}$ and $\mathbb{Q}_{p}$ denote the ring of $p$-adic integers and the field of $p$-adic numbers respectively.

Lemma 3.1. Let $p$ be a prime. For any $n \in \mathbb{N}$ and $x \in \mathbb{Z}_{p},\left(\begin{array}{l}x \\ n\end{array}\right)$ is p-integral.

Proof. We may choose $x^{\prime} \in \mathbb{N}$ such that

$$
x \equiv x^{\prime}\left(\bmod p^{\operatorname{ord}_{p}(n !)+1}\right) .
$$

Then

$$
\begin{aligned}
\left(\begin{array}{l}
x \\
n
\end{array}\right) & =\frac{x(x-1) \cdots(x-n+1)}{n !} \\
& \equiv \frac{x^{\prime}\left(x^{\prime}-1\right) \cdots\left(x^{\prime}-n+1\right)}{n !}=\left(\begin{array}{l}
x^{\prime} \\
n
\end{array}\right)(\bmod p) .
\end{aligned}
$$

This shows that $\left(\begin{array}{l}x \\ n\end{array}\right) \in \mathbb{Z}_{p}$ since $\left(\begin{array}{l}x^{\prime} \\ n\end{array}\right) \in \mathbb{Z}$. 
Proof of (1.10). Let $\omega$ be the Teichmüller character of the multiplicative group $(\mathbb{Z} / p \mathbb{Z})^{*}$. As an application of Hensel's lemma, we know that

$$
\omega(a) \in \mathbb{Z}_{p}, \quad a=1, \ldots, p-1,
$$

are exactly all $(p-1)$ th roots of unity in $\mathbb{Q}_{p}$. Moreover, $\omega(g)$ is a primitive $(p-1)$ th root of unity if and only if $g$ is a primitive root modulo $p$. Let $\varpi \in \mathbb{Z}_{p}$ be an arbitrary primitive $(p-1)$ th root of unity in $\mathbb{Q}_{p}$. Then

$$
\begin{aligned}
\sum_{k \equiv r(\bmod p-1)} s(n, k) S(k, m) a^{k} & =\sum_{k=0}^{n} s(n, k) S(k, m) a^{k} \cdot \frac{1}{p-1} \sum_{j=1}^{p-1} \varpi^{j(k-r)} \\
& =\frac{1}{p-1} \sum_{j=1}^{p-1} \varpi^{-j r} \sum_{k} s(n, k) S(k, m)\left(a \varpi^{j}\right)^{k} .
\end{aligned}
$$

It is known that

$$
m ! S(k, m)=\sum_{i=0}^{m}\left(\begin{array}{c}
m \\
i
\end{array}\right)(-1)^{m-i} i^{k}
$$

so

$$
\begin{aligned}
\sum_{k \equiv r(\bmod p-1)} & s(n, k) S(k, m) a^{k} \\
= & \frac{1}{p-1} \sum_{j=1}^{p-1} \varpi^{-j r} \sum_{k} s(n, k)\left(\frac{1}{m !} \sum_{i=0}^{m}\left(\begin{array}{c}
m \\
i
\end{array}\right)(-1)^{m-i} i^{k}\right)\left(a \varpi^{j}\right)^{k} \\
= & \frac{1}{m !(p-1)} \sum_{j=1}^{p-1} \varpi^{-j r} \sum_{i=0}^{m}\left(\begin{array}{c}
m \\
i
\end{array}\right)(-1)^{m-i} \sum_{k} s(n, k)\left(a i \varpi^{j}\right)^{k} \\
= & \frac{n !}{m !(p-1)} \sum_{j=1}^{p-1} \varpi^{-j r} \sum_{i=0}^{m}\left(\begin{array}{c}
m \\
i
\end{array}\right)(-1)^{m-i}\left(\begin{array}{c}
a i \varpi^{j}+n-1 \\
n
\end{array}\right) .
\end{aligned}
$$

Thus applying Lemma 3.1, it is derived that

$$
\operatorname{ord}_{p}\left(\sum_{k \equiv r(\bmod p-1)} s(n, k) S(k, m) a^{k}\right) \geq \operatorname{ord}_{p}(n ! / m !)=\operatorname{ord}_{p}(n !)-\operatorname{ord}_{p}(m !) \text {. }
$$

Lemma 3.2. Let $n$ and $l$ be positive integers. Then

$$
(n-i)\left(\begin{array}{c}
i \\
l-1
\end{array}\right) \leq\left(\begin{array}{l}
n \\
l
\end{array}\right) \quad \text { for each integer } 0 \leq i \leq n .
$$

Proof. Clearly, the desired result is true if $n<l$. Below we assume that $n \geq l$. It is easy to check that

$$
(n-i)\left(\begin{array}{c}
i \\
l-1
\end{array}\right) \geq(n-i+1)\left(\begin{array}{l}
i-1 \\
l-1
\end{array}\right) \Leftrightarrow i \leq \frac{(l-1)(n+1)}{l} .
$$


Hence

$$
\begin{aligned}
(n-i)\left(\begin{array}{c}
i \\
l-1
\end{array}\right) & \leq(n-\lfloor(l-1)(n+1) / l\rfloor)\left(\begin{array}{c}
\lfloor(l-1)(n+1) / l\rfloor \\
l-1
\end{array}\right) \\
& =\left\lceil\frac{n-l+1}{l}\right\rceil\left(\begin{array}{c}
n-\lceil(n-l+1) / l\rceil \\
l-1
\end{array}\right) \\
& \leq \frac{n}{l}\left(\begin{array}{c}
n-1 \\
l-1
\end{array}\right)=\left(\begin{array}{c}
n \\
l
\end{array}\right) .
\end{aligned}
$$

Proof of (1.11). We use induction on $\operatorname{deg} f$. The case $\operatorname{deg} f=0$ follows from (1.10) by setting $m=1$. Below we assume that $\operatorname{deg} f>0$ and (1.11) holds for smaller values of $\operatorname{deg} f$. It is known (cf. [1, p. 215]) that

$$
k s(n, k)=\sum_{i=k-1}^{n-1}\left(\begin{array}{c}
n \\
i
\end{array}\right)(n-i-1) ! s(i, k-1) \quad(k \geq 1) .
$$

Write $f(x)=x f_{1}(x)+c$ with $\operatorname{deg} f_{1}=\operatorname{deg} f-1$. Then

$$
\begin{aligned}
& \sum_{k \equiv r(\bmod p-1)} s(n, k) f(k) a^{k} \\
= & \sum_{k \equiv r(\bmod p-1)} f_{1}(k) a^{k} \sum_{i=k-1}^{n-1}\left(\begin{array}{c}
n \\
i
\end{array}\right)(n-i-1) ! s(i, k-1) \\
& +c \sum_{k \equiv r(\bmod p-1)} s(n, k) a^{k} \\
= & \sum_{i=0}^{n-1} \frac{n !}{i !(n-i)} \sum_{k \equiv r(\bmod p-1)} s(i, k-1) f_{1}(k) a^{k}+c \sum_{k \equiv r(\bmod p-1)} s(n, k) a^{k} \\
= & \sum_{i=0}^{n-1} \frac{n ! a}{i !(n-i)} \sum_{k \equiv r-1(\bmod p-1)} s(i, k) f_{1}(k+1) a^{k}+c \sum_{k \equiv r(\bmod p-1)} s(n, k) a^{k} .
\end{aligned}
$$

When $i=0$,

$$
\begin{aligned}
\operatorname{ord}_{p}\left(\frac{a n !}{0 !(n-0)} \sum_{k \equiv r-1}(\bmod p-1)\right. & \left.s(0, k) f_{1}(k+1) a^{k}\right) \\
& \geq \operatorname{ord}_{p}(n !)-\operatorname{ord}_{p}(n) \\
& \geq \begin{cases}0=\operatorname{ord}_{p}(n !)-\log _{p}\left(\begin{array}{l}
n \\
0
\end{array}\right) & \text { if } n<p, \\
\operatorname{ord}_{p}(n !)-\log _{p} n \geq \operatorname{ord}_{p}(n !)-\log _{p}\left(\begin{array}{l}
n \\
l
\end{array}\right) & \text { otherwise. }\end{cases}
\end{aligned}
$$


For every $0<i \leq n-1$, by the induction hypothesis,

$$
\begin{gathered}
\operatorname{ord}_{p}\left(\frac{n ! a}{i !(n-i)} \sum_{k \equiv r-1(\bmod p-1)} s(i, k) f_{1}(k+1) a^{k}\right) \\
\geq \operatorname{ord}_{p}(n !)-\operatorname{ord}_{p}(i !)-\operatorname{ord}_{p}(n-i)+\operatorname{ord}_{p}(i !)-\log _{p}\left(\begin{array}{c}
i \\
l^{\prime}
\end{array}\right) \\
\quad=\operatorname{ord}_{p}(n !)-\operatorname{ord}_{p}(n-i)-\log _{p}\left(\begin{array}{c}
i \\
l^{\prime}
\end{array}\right)
\end{gathered}
$$

where $l^{\prime}=\min \{\operatorname{deg} f-1,\lfloor i / p\rfloor\}$. It suffices to show that

$$
\operatorname{ord}_{p}(n-i)+\log _{p}\left(\begin{array}{l}
i \\
l^{\prime}
\end{array}\right) \leq \log _{p}\left(\begin{array}{l}
n \\
l
\end{array}\right) \text {. }
$$

When $i>n-p$, clearly $l-1 \leq l^{\prime} \leq l$ and $\operatorname{ord}_{p}(n-i)=0$. Hence

$$
\left(\begin{array}{c}
i \\
l^{\prime}
\end{array}\right) \leq \max \left\{\left(\begin{array}{c}
n-1 \\
l-1
\end{array}\right),\left(\begin{array}{c}
n-1 \\
l
\end{array}\right)\right\} \leq\left(\begin{array}{c}
n \\
l
\end{array}\right)
$$

Below we assume that $i \leq n-p$. Then $\lfloor i / p\rfloor+1 \leq\lfloor n / p\rfloor$. If $\operatorname{deg} f-1 \leq\lfloor i / p\rfloor$, then applying Lemma 3.2 we obtain

$$
(n-i)\left(\begin{array}{c}
i \\
l^{\prime}
\end{array}\right)=(n-i)\left(\begin{array}{c}
i \\
\operatorname{deg} f-1
\end{array}\right) \leq\left(\begin{array}{c}
n \\
\operatorname{deg} f
\end{array}\right)=\left(\begin{array}{l}
n \\
l
\end{array}\right)
$$

since $\operatorname{deg} f \leq\lfloor n / p\rfloor$ now. Also, when $\lfloor i / p\rfloor<\operatorname{deg} f-1$, we have

$$
(n-i)\left(\begin{array}{c}
i \\
l^{\prime}
\end{array}\right)=(n-i)\left(\begin{array}{c}
i \\
\lfloor i / p\rfloor
\end{array}\right) \leq\left(\begin{array}{c}
n \\
\lfloor i / p\rfloor+1
\end{array}\right) \leq\left(\begin{array}{c}
n \\
l
\end{array}\right)
$$

since $\lfloor i / p\rfloor+1 \leq \min \{\operatorname{deg} f,\lfloor n / p\rfloor\}=l$. In each of the above two cases, we obtain

$$
\operatorname{ord}_{p}(n-i)+\log _{p}\left(\begin{array}{c}
i \\
l^{\prime}
\end{array}\right) \leq \log _{p}(n-i)+\log _{p}\left(\begin{array}{l}
i \\
l^{\prime}
\end{array}\right) \leq \log _{p}\left(\begin{array}{l}
n \\
l
\end{array}\right)
$$

4. Congruences for Stirling numbers: $k \equiv r\left(\bmod p^{\alpha}(p-1)\right)$. In this section, we shall prove Theorem 1.3. Define

$$
C_{d, r}(n, m, a)=\sum_{k \equiv r(\bmod d)} s(n, k) S(k, m) a^{k} .
$$

Let $\zeta_{d}$ be a primitive $d$ th root of the unity. Then 


$$
\begin{aligned}
C_{d, r}(n, m, a) & \\
& =\sum_{k} s(n, k) a^{k}\left(\frac{1}{m !} \sum_{i=0}^{m}\left(\begin{array}{c}
m \\
i
\end{array}\right)(-1)^{m-i} i^{k}\right)\left(\frac{1}{d} \sum_{j=0}^{d-1} \zeta_{d}^{j(k-r)}\right) \\
& =\frac{1}{m ! d} \sum_{j=0}^{d-1} \zeta_{d}^{-j r} \sum_{i=0}^{m}\left(\begin{array}{c}
m \\
i
\end{array}\right)(-1)^{m-i} \sum_{k} s(n, k)\left(a i \zeta_{d}^{j}\right)^{k} \\
& =\frac{(-1)^{n}}{m ! d} \sum_{i=0}^{m}\left(\begin{array}{c}
m \\
i
\end{array}\right)(-1)^{m-i} \sum_{j=0}^{d-1} \zeta_{d}^{-j r}\left(-a i \zeta_{d}^{j}\right)_{n},
\end{aligned}
$$

where

$(x)_{0}=1 \quad$ and $\quad(x)_{k}=x(x-1) \cdots(x-k+1) \quad$ for $k=1,2, \ldots$

Lemma 4.1. Let $p$ be a prime and $\alpha$ be a positive integer. Then for any $1 \leq k \leq p^{\alpha}(p-1)$, we have

$$
s\left(p^{\alpha}(p-1), k\right) \equiv \begin{cases}1(\bmod p) & \text { if } k \equiv 0\left(\bmod p^{\alpha-1}(p-1)\right), \\ 0(\bmod p) & \text { otherwise. }\end{cases}
$$

Proof. Let $x$ be a variable. Apparently

Thus

$$
x(x+1) \cdots(x+p-1) \equiv x^{p}-x(\bmod p) .
$$

$$
\begin{aligned}
\sum_{k=0}^{p^{\alpha}(p-1)} s\left(p^{\alpha}(p-1), k\right) x^{k}=x(x+1) \cdots\left(x+p^{\alpha}(p-1)-1\right) \\
\equiv(x(x+1) \cdots(x+p-1))^{p^{\alpha-1}(p-1)} \equiv\left(x^{p}-x\right)^{p^{\alpha-1}(p-1)} \\
=\sum_{j=0}^{p^{\alpha-1}(p-1)}\left(\begin{array}{c}
p^{\alpha-1}(p-1) \\
j
\end{array}\right)(-1)^{j} x^{p^{\alpha}(p-1)-(p-1) j}(\bmod p) .
\end{aligned}
$$

By the Lucas congruence, we know that for $0 \leq j \leq p^{\alpha-1}(p-1)$,

$$
\left(\begin{array}{cc}
p^{\alpha-1}(p-1) \\
j
\end{array}\right) \equiv \begin{cases}\left(\begin{array}{c}
p-1 \\
j / p^{\alpha-1}
\end{array}\right)(\bmod p) & \text { if } p^{\alpha-1} \mid j \\
0(\bmod p) & \text { otherwise }\end{cases}
$$

Hence

$$
\begin{aligned}
\sum_{k=0}^{p^{\alpha}(p-1)} s\left(p^{\alpha}(p-1), k\right) x^{k} & \equiv \sum_{j=0}^{p-1}\left(\begin{array}{c}
p-1 \\
j
\end{array}\right)(-1)^{j} x^{p^{\alpha}(p-1)-p^{\alpha-1}(p-1) j} \\
& \equiv \sum_{j=0}^{p-1} x^{p^{\alpha-1}(p-1)(p-j)}=\sum_{j=1}^{p} x^{p^{\alpha-1}(p-1) j}(\bmod p),
\end{aligned}
$$

which is evidently equivalent to (4.2). 
Proof of Theorem 1.3. Since

$s(n+1, k)=n s(n, k)+s(n, k-1) \quad$ and $\quad S(n+1, k)=k S(n, k)+S(n, k-1)$,

we have

$$
\begin{aligned}
\sum_{k \equiv r(\bmod d)} s(n+1, k) S(k, m) a^{k} & \\
= & \sum_{k \equiv r(\bmod d)} s(n, k) S(k, m) a^{k}+\sum_{k \equiv r(\bmod d)} s(n, k-1) S(k, m) a^{k} \\
= & n \sum_{k \equiv r(\bmod d)} s(n, k) S(k, m) a^{k} \\
& +\sum_{k \equiv r-1(\bmod d)} s(n, k)(m S(k, m)+S(k, m-1)) a^{k+1},
\end{aligned}
$$

that is,

$$
\begin{aligned}
& C_{d, r}(n+1, m, a) \\
& \quad=n C_{d, r}(n, m, a)+a m C_{d, r-1}(n, m, a)+a C_{d, r-1}(n, m-1, a) .
\end{aligned}
$$

Also observe that

$$
\left\lfloor\frac{n-p^{\alpha}}{p^{\alpha}(p-1)}\right\rfloor=\left\lfloor\frac{\left\lfloor n / p^{\alpha}\right\rfloor-1}{p-1}\right\rfloor .
$$

Without loss of generality, we may assume that $p^{\alpha}$ divides $n$. We reason by induction on $n$. Clearly the case $n<p^{\alpha+1}$ is trivial. Let $\zeta$ be a primitive $p^{\alpha}(p-1)$ th root of unity. Then in view of (4.1),

$$
\begin{aligned}
& (-1)^{n} m ! p^{\alpha}(p-1) C_{p^{\alpha}(p-1), r}\left(n+p^{\alpha}(p-1), m, a\right) \\
& =\sum_{i=0}^{m}\left(\begin{array}{c}
m \\
i
\end{array}\right)(-1)^{m-i} \sum_{j=1}^{p^{\alpha}(p-1)} \zeta^{-j r}\left(-a i \zeta^{j}\right)_{n}\left(-a i \zeta^{j}-n\right)_{p^{\alpha}(p-1)} \\
& =\sum_{i=0}^{m}\left(\begin{array}{c}
m \\
i
\end{array}\right)(-1)^{m-i} \sum_{j=1}^{p^{\alpha}(p-1)} \zeta^{-j r}\left(-a i \zeta^{j}\right)_{n} \sum_{k=0}^{p^{\alpha}(p-1)} s\left(p^{\alpha}(p-1), k\right)\left(a i \zeta^{j}+n\right)^{k} \\
& =\sum_{i=0}^{m}\left(\begin{array}{c}
m \\
i
\end{array}\right)(-1)^{m-i} \sum_{j=1}^{p^{\alpha}(p-1)} \zeta^{-j r}\left(-a i \zeta^{j}\right)_{n} \\
& \quad \times \sum_{k=0}^{p^{\alpha}(p-1)} s\left(p^{\alpha}(p-1), k\right) \sum_{l=0}^{k}\left(\begin{array}{c}
k \\
l
\end{array}\right)\left(a i \zeta^{j}\right)^{l} n^{k-l} .
\end{aligned}
$$


Applying Lemma 4.1, we have

$$
\begin{aligned}
& m ! C_{p^{\alpha}(p-1),}\left(n+p^{\alpha}(p-1), m, a\right) \sum_{i=0}^{m}\left(\begin{array}{c}
m \\
i
\end{array}\right)(-1)^{m-i} \sum_{k=0}^{p^{\alpha}(p-1)} s\left(p^{\alpha}(p-1), k\right) \\
&= \sum_{l=0}^{k}\left(\begin{array}{c}
k \\
l
\end{array}\right)(a i)^{l} n^{k-l} \cdot \frac{\sum_{j=1}^{p^{\alpha}(p-1)} \zeta^{j(l-r)}\left(-a i \zeta^{j}\right)_{n}}{(-1)^{n} p^{\alpha}(p-1)} \\
&= \sum_{i=0}^{m}\left(\begin{array}{c}
m \\
i
\end{array}\right)(-1)^{m-i} \sum_{k=0}^{p^{\alpha}(p-1)} s\left(p^{\alpha}(p-1), k\right) \\
& \times \sum_{l=0}^{k}\left(\begin{array}{c}
k \\
l
\end{array}\right)(a i)^{l} n^{k-l} C_{p^{\alpha}(p-1), r-l}(n, 1, a i) \\
& \equiv \sum_{i=0}^{m}\left(\begin{array}{c}
m \\
i
\end{array}\right)(-1)^{m-i} \sum_{k=1}^{p}(a i)^{k p^{\alpha-1}(p-1)} C_{p^{\alpha}(p-1), r-k p^{\alpha-1}(p-1)}(n, 1, a i) \\
& \quad\left(\bmod p^{\left\lfloor\frac{n-p^{\alpha}}{p^{\alpha}(p-1)}\right\rfloor+1}\right),
\end{aligned}
$$

since $p^{\alpha} \mid n$ and

$$
C_{p^{\alpha}(p-1), r-l}(n, 1, a i) \equiv 0\left(\bmod p^{\left\lfloor\frac{n-p^{\alpha}}{p^{\alpha}(p-1)}\right\rfloor}\right)
$$

by the induction hypothesis on $n$. When $p \mid a$, clearly

$$
m ! C_{p^{\alpha}(p-1), r}\left(n+p^{\alpha}(p-1), m, a\right) \equiv 0\left(\bmod p^{\left\lfloor\frac{n-p^{\alpha}}{p^{\alpha}(p-1)}\right\rfloor+1}\right) .
$$

If $p \nmid a$,

$$
\begin{aligned}
& m ! C_{p^{\alpha}(p-1), r}\left(n+p^{\alpha}(p-1), m, a\right) \\
& \equiv \sum_{\substack{0 \leq i \leq m \\
p \nmid i}}\left(\begin{array}{c}
m \\
i
\end{array}\right)(-1)^{m-i} \sum_{k=1}^{p}\left((a i)^{p-1}\right)^{k p^{\alpha-1}} C_{p^{\alpha}(p-1), r-k p^{\alpha-1}(p-1)}(n, 1, a i) \\
& \equiv \sum_{\substack{0 \leq i \leq m \\
p \nmid i}}\left(\begin{array}{c}
m \\
i
\end{array}\right)(-1)^{m-i} \sum_{k=1}^{p} C_{p^{\alpha}(p-1), r-k p^{\alpha-1}(p-1)}(n, 1, a i)\left(\bmod p^{\left\lfloor\frac{n-p^{\alpha}}{p^{\alpha}(p-1)}\right\rfloor+1}\right) .
\end{aligned}
$$

Now

$$
\begin{gathered}
\sum_{k=1}^{p} C_{p^{\alpha}(p-1), r-k p^{\alpha-1}(p-1)}(n, 1, a i)=\sum_{k=1}^{p} \sum_{\substack{l \equiv r-k p^{\alpha-1}(p-1) \\
\left(\bmod p^{\alpha}(p-1)\right)}} s(n, l)(a i)^{l} \\
=\sum_{l \equiv r\left(\bmod p^{\alpha-1}(p-1)\right)} s(n, l)(a i)^{l}=C_{p^{\alpha-1}(p-1), r}(n, 1, a i) .
\end{gathered}
$$


Thus it suffices to show that

$$
\operatorname{ord}_{p}\left(C_{p^{\alpha-1}(p-1), r}(n, 1, a i)\right) \geq\left\lfloor\frac{n-p^{\alpha}}{p^{\alpha}(p-1)}\right\rfloor+1 .
$$

Note that $n \geq p^{\alpha}$ now. If $\alpha=1$, then by (1.10),

$\operatorname{ord}_{p}\left(C_{p-1, r}(n, 1, a i)\right) \geq \operatorname{ord}_{p}(n !) \geq\left\lfloor\frac{n}{p}\right\rfloor \geq\left\lfloor\frac{n+p(p-2)}{p(p-1)}\right\rfloor=\left\lfloor\frac{n-p}{p(p-1)}\right\rfloor+1$.

Also if $\alpha \geq 2$, by the induction hypothesis, we have

$$
\operatorname{ord}_{p}\left(C_{p^{\alpha-1}(p-1), r}(n, 1, a i)\right) \geq\left\lfloor\frac{n-p^{\alpha-1}}{p^{\alpha-1}(p-1)}\right\rfloor \geq\left\lfloor\frac{n-p^{\alpha}}{p^{\alpha}(p-1)}\right\rfloor+1 \text {. }
$$

Acknowledgements. We thank our advisor, Professor Zhi-Wei Sun, for his helpful suggestions on this paper.

\section{References}

[1] L. Comtet, Advanced Combinatorics, Reidel, Dordrecht, 1974.

[2] D. M. Davis and Z. W. Sun, A number-theoretic approach to homotopy exponents of $S U(n)$, J. Pure Appl. Algebra 209 (2007), 57-69.

[3] L. E. Dickson, History of the Theory of Numbers, Vol. I, Chelsea, New York, 1966.

[4] R. L. Graham, D. E. Knuth and O. Patashnik, Concrete Mathematics, 2nd ed., Addison-Wesley, Reading, MA, 1994.

[5] J. H. van Lint and R. M. Wilson, A Course in Combinatorics, 2nd ed., Cambridge Univ. Press, Cambridge, 2001.

[6] J. Sándor and B. Crstici, Handbook of Number Theory II, Kluwer, Dordrecht, 2004.

[7] Z. W. Sun, Polynomial extension of Fleck's congruence, Acta Arith. 122 (2006), 91-100.

[8] -, Combinatorial congruences and Stirling numbers, ibid. 126 (2007), 387-398.

[9] - Fleck quotients and Bernoulli numbers, preprint, arXiv:math.NT/0608328.

[10] Z. W. Sun and D. M. Davis, Combinatorial congruences modulo prime powers, Trans. Amer. Math. Soc. 359 (2007), 5525-5553.

[11] Z. W. Sun and D. Wan, Lucas type congruences for cyclotomic $\psi$-coefficients, Int. J. Number Theory 4 (2008), 155-170.

[12] -, -, On Fleck quotients, Acta Arith. 127 (2007), 337-363.

[13] D. Wan, Combinatorial congruences and $\psi$-operators, Finite Fields Appl. 12 (2006), 693-703.

[14] C. S. Weisman, Some congruences for binomial coefficients, Michigan Math. J. 24 (1977), 141-151.

Hui-Qin Cao

Department of Applied Mathematics

Nanjing Audit University

Nanjing 210029

People's Republic of China

E-mail: caohq@nau.edu.cn
Hao Pan

Department of Mathematics

Nanjing University

Nanjing 210093

People's Republic of China

E-mail: haopan79@yahoo.com.cn 